\title{
Loneliness and How Group Visits Help People Heal
}

\section{Jeffrey S. Geller, MD, FP}

\begin{abstract}
Jeffrey S. Geller, MD, FP, is an award-winning family physician and integrative medicine practitioner who has invested his career, passion, and energy in creative initiatives that are changing the landscape of medicine. In a field that is often preoccupied with ordering tests and procedures, Dr. Geller offers a paradigm shift in medicine by emphasizing patients' personal states of well-being and assessing their social support structures. An expert in community group visits, Dr. Geller discusses the impact of loneliness on health and how empowerment groups can help people heal.
\end{abstract}

\section{Q: How common is loneliness today in society?}

Jeffrey S. Geller, MD, FP: Based on the data that we have today, clinicians can expect that $20 \%-30 \%$ of people are lonely, and the lifetime incidence of loneliness is about $80 \%$ that is, people feeling lonely at some point during their life. However, it is important to point out that loneliness is different than depression or happiness because it is a perception. For example, when clinicians see a person for depression the presentation is often fairly clear, and there is a code for the diagnosis. As a result, clinicians bill for depression and check off the box that this patient is depressed. Loneliness, on the other hand, is not billable by psychiatrists or other clinicians and it is not as easy to assess “Are people lonely or not?" Typically, researchers examine medical records to determine how many people are depressed and what percentage of these people are taking medication, and so on, but until recently these records were not available for measuring loneliness.

We also know that loneliness varies depending on a person's age. Older people tend to be less lonely than younger people, which is a surprise to many. In fact, a recent Cigna survey, called the 2020 Loneliness Index, included more than 10,000 adults ages 18 and over, and results showed that three in five Americans reported feeling lonely, men may be lonelier than women, and younger people ages $18-22$ years tend to be lonelier than people over the age of $72 .^{1}$

Older people tend to be more socially isolated, which sounds similar to loneliness, but it is a different issue. When someone is socially isolated, he or she may still know that there are people such as their children who love and understand them, so loneliness is not the predominant feeling. Younger people, however, experience many types of emotional, physical, and social changes for the first time, which can leave them especially vulnerable to feeling lonely. Teens often do not think that their parents understand them and maybe they do not think their friends understand them. So that is what creates loneliness - a feeling that someone does not understand you. At this fragile age, people may not know who they are, so they wonder how can anyone else know who they are either.

\section{Q: How does loneliness impact a person's health?}

Dr. Geller: Most of the studies regarding loneliness are associational studies, and such studies have shown that loneliness is definitely linked to negative health outcomes. For example, we know that people who are lonely are five times more likely to experience a heart attack and five times more likely to die within a five-year period than people who are not lonely. It is possible that multiple factors contribute to this. Compared with people who are not lonely, people who are lonely may smoke more cigarettes or engage in less self-care. A biologic component may also contribute to poor health outcomes. For example, loneliness leads to increased stress, which triggers the axis of hormones that can raise blood pressure, affect sleeping patterns, and lead to worse health outcomes overall. It is similar with depression, where a person is far more likely to have poor health outcomes compared with someone who is not depressed.

One of the questions that I have pondered throughout the years is as follows: Are lonely people going to the doctor more often because they are self-treating their loneliness, or are they truly more ill than people who are not lonely? In one of the first studies I conducted, results showed that lonely people were not sicker, but rather they were using health care facilities more often. ${ }^{2}$ According to prior research, compared with non-lonely people, people who are lonely are two to three times more likely to use the emergency room. In fact, my colleagues and I discovered that people were using our health center six times more often if they were lonely.

It is important to point out that a person typically does not come to a health center and say, "I am lonely. I need to talk 
with someone." They come to the health center and they are diagnosed with a medical illness. The bottom line is that being lonely is an indicator of poorer health, and insurance companies have picked up on this and tend to screen for loneliness more frequently than physicians.

Loneliness may be missed or overlooked by clinicians and yet it is a predictor of hospital emergency room use and can be a costly health risk factor. ${ }^{2,3}$ If a person is using the emergency room or health center three times as often as the next person, that is a lot of money being spent, so loneliness becomes expensive within the health care system.

Q: What specific factors contribute to loneliness in our world today and when should clinicians be on the lookout for loneliness in their patient?

Dr. Geller: Most importantly, loneliness is often tied to the feeling that there is no one there who understands you. There are two parts to this. For example, a person may not have any friends, but they enjoy being alone and do not feel lonely. On the other hand, another person might have 100 friends, and yet this person feels that none of their friends truly understand who they are. Therefore, even if this person is surrounded by their friends and everyone agrees that they like each other, this person still feels lonely. In this example, people understand others, but that person does not believe that others understand them. This perception can be related to personality, bonding, and cultural issues, and a person may not have the tools to build close connections with people.

Another example is that a person can feel that they are different within their community or circumstances. This may be because they think differently, look differently, or are of a different race, religion, or sexual orientation. This can lead people to feel that no one really understands them and can make them feel lonely.

I want to mention another important point about loneliness. Depression by and large does not happen that quickly. A person does not typically wake up one day and become depressed. There is usually a series of events that lead to a state of depression. But with loneliness, there can be suddenness to it more than other conditions. So I refer to this as relative loneliness, and probably everyone has experienced relative loneliness at some point in their lives. For example, a person may suddenly not feel comfortable talking with someone about something and that causes them to feel lonely. Or a person feels alone with a particular problem, so it is relative to that problem. Relative loneliness could happen during the course of a day. This type of loneliness is common and prevalent and may be brought on when a person, for example, learned that someone they love is ill, or they realized they made a mistake at work, or they received a bad grade in school.

Clinicians should be on the lookout for these various types of loneliness when talking with their patients and also pay attention to people who come in for visits more often than other patients, but who are not necessarily sicker than other people coming to the office. Then, it is also important to simply ask all patients about their social support structure.

Q: How should a clinician assess for loneliness in their patients?

Dr. Geller: There is an inventory called the UCLA Loneliness Questionnaire, which most people use as the main standard for measuring loneliness, and there are several versions. The questionnaire includes about 20 questions, including "Do you feel alone?" and "Do you feel that people understand you?" But there is one main question that appears to have the highest association with loneliness, and that question is, "Do you feel that there is someone in your life who really understands you?"

When I meet a patient for the first time, I usually say, "Before we start talking about your medical issues, can you please tell me a little bit about who you are?" My questions then begin with, "Who are you living with? Do these people understand you? Are your parents alive? What is your relationship like with your parents? Do you have any siblings or friends who you feel close with that you talk with every day?"

I take a detailed social and support history. If I get a lot of negative answers, then I want to know more and might ask, "You say you live alone? Why is that? Is that by choice or not?" I start thinking that a person may be at risk for being lonely. Then I say, "Okay, now tell me about your problem and why you came here today." The patient may then say, "I have headaches." I then will ask, "Do you have anyone you talk to about your headaches?" and I begin to tease out the problem. I usually do that right up front in my visits. It is not totally infrequent that the patient also recognizes that this is a different line of questioning, and they might say, "Well, what does it matter if someone knows if I have a headache?" So, then I get to respond, "Well, you know, if something were to happen to you, I would want to know that there is someone there who you can talk with or who can help take care of you."

Whenever I am seeing a patient for the first time I also go right into the question, "Do you feel that there is anyone who understands you?" If someone is feeling very depressed and has recently lost their mother and is visiting the office frequently, I might say, "Is there anyone else you know who has lost a mother who is similar to you and is not doing so well?" For other types of problems I might ask, "Have you met anyone else with back pain?" or "Do you know anyone else with high blood pressure?"

As clinicians, we realize that making a diagnosis is fairly simple much of the time, and in medical school that is all we thought about as students - making the diagnosis. The thing about that is it can keep a clinician from focusing on developing a good relationship with the patient. If a clinician has a good relationship with the patient, the patient will keep coming back, and they will give you more clues about who they really are.

Common problems have algorithms for treatments that are commonly known or searchable, but the real medicine is how a clinician helps a person in the context of who they are to feel better and feel like they could be productive in the world and 
connected to other people. That is why I take the approach I do right from the onset. It is about the art of communication - if someone likes you, they are more likely to listen to you. We spend our time talking as clinicians and say, "Oh, you need to exercise more." But then they leave, and they do not exercise more.

I put effort into having my patients like me and work on building trust. I might even say to a patient who is feeling lonely, "I am rooting for you. It sounds like you have had a rough time, and I want you to get better and get back to work. Maybe we can make that one of our goals." This then leads into a discussion about attending group visits to achieve that goal and to help this person meet and connect with other people.

Q: Tell us about the group visits that you created to address loneliness and its relation to health problems.

Dr. Geller: After the assessment process that I just described, I am in a unique situation to be able to send people with medical problems to a group visit. That group may be a diabetes group, a yoga group, and so on. I say to the patient, "I am going to have you attend a group with other people with diabetes because I think it might be a better way for you to receive care."

The first group I started was a diabetes group. Initially, I grouped people by illness or condition, so there was a cardiac group, a pain group, and prenatal groups. We then started adding groups for integrative medicine such as acupuncture and osteopathy groups. Then I slowly started uncoupling the disease from the group, and my colleagues and I decided to have an exercise group and determine who best would benefit from this group. Such a group might include a variety of people such as someone who has diabetes, or who is overweight, or who is depressed. The groups started to become less about illness and more about what a person needed in order to feel well.

Then we took the groups to another level to build connections within the groups, and we had the groups create projects. We had a group of people with developmental delay, and they created a dance and exercise routine that became a videotape. This was the second generation of groups where we started to really learn how to bring people together, and now we call them "empowerment groups." In these empowerment groups we would ask the people in the group, "What do you think you need to feel better?" In one of the groups, we would exercise together and then after about a year or two, the group bonded, and they decided they wanted to go on a fall foliage field trip together. We learned that doing projects together, particularly projects that are new and involve planning, brings a higher level of connection between people.

It is one thing to have people passively attend a group; however, it is another thing for the group to determine how they will pay for the fall foliage trip, when they will leave, what form of transportation they will use, and so on. It turns out that this kind of group planning and creating is essential for building relationships. People may have friends who they feel very close to who are very different than them, but it is because they have gone through something together that they build trust and understanding.
I remember the first fall foliage trip. It was raining and we were wearing plastic garbage bags, and we were driving up to see the foliage, and it was still quite beautiful, and there were waterfalls. A lot of people felt, "Oh, this reminds me of home." To this day, 20 years after that first trip, people say, "Remember when we went to see the fall leaves and it was raining, and we went to the waterfall, and we took pictures?" and that creates relationships. People are getting a lot more value and connection with others in group activities.

Currently, I am creating a center offering mainly primary care group visits - that is the degree to which I believe in this model and the transformation of medicine. My physicals are done in group visits for adults and children. If someone has a headache, they attend the headache group. If someone needs a refill, they attend a refill group. If they are working on their diet, they attend a refill group with dietary discussions. We have a yoga group, massage group, and osteopathic manipulation group. So if a person has pain, not only do they come in to get a refill and a referral, but they can also talk to other people and get a massage.

It is important to point out that I never end the groups. I find that by having an open-ended group model where people can come, they often will learn not only the curriculum, but they will learn what other people have done in the community to succeed, and they will have a reduction in loneliness. An ongoing group is part of the treatment and creates community for people. Also, there are different participation levels within a group. A group can accommodate people who need support and want to be there every day, or it can work for someone who wants to drop in and participate as little as possible, but still have their health care needs met. It is a very effective model.

Q: What are some of the significant outcomes you have seen as a result of this group visit model?

Dr. Geller: Over the years, my colleagues and I have published a number of articles regarding the impact of the working group visits. Our most successful program to date is called the Pediatric Obesity Empowerment Model (POEM). We conducted a research study with the POEM groups for three years, and the study included more than 400 children. $^{4}$ Results of our research showed that $62 \%$ of the participants over those three years lost weight.

In our community and in the world, pediatric obesity is a very challenging issue, so it was hopeful to see that our project was extremely successful. We found that stress reduction was the number one most important factor for weight loss among these groups. In these groups, we did some exercise and we talked about food, for example. The second most important factor related to successful weight loss was reducing sugary beverages.

We asked the children, "What do you need to do to be healthier?" So we played, went apple picking, and did lots of activities. We had an exercise instructor the children really liked, and we did activities that the children chose. They were empowered and they felt like they had a home. It was a class for 


\section{To Contact Dr. Jeffrey S. Geller}

Jeffrey S. Geller, MD, FP

Integrated Center for Group Medical Visits (ICGMV)

Lawrence, Massachusetts

Website: https://icgmv.org

everybody and their stress reduced. When their stress reduced, they lost weight.

We have also published data on our chronic pain groups and health-related quality of life improved significantly in the participants of those groups. Our group for people with developmental delay, which is perhaps one of the most unempowered subsegments of our population, was in a weight loss group, and the average participant lost six pounds for a year and a half, which, again, is very significant.

Q: What do you want to say to other clinicians about loneliness and the impact of group visits with patients?

Dr. Geller: I want to encourage clinicians to take a moment and to really listen to their patients. Find something you like about them so you can connect with them. If they are alone or lonely, then focus on why they are alone and what they may need to learn so that they can build strong relationships with others.

I also want to mention that on the Integrated Center for Group Medical Visits (https://icgmv.org), we have resources for clinicians to learn more about starting their own groups. If clinicians can offer groups in their practice, they do not need a huge space. They can utilize a waiting room or some area where they can bring people together so they can connect. It is very rewarding to see people's health improve, not only because of the focus on their health, but also because we are now focusing on their social situation. I personally believe that having this focus, one significantly reduces the chances of dying more than any prescribed medication a clinician could use.

\section{References}

1. Cigna. Cigna Takes Action to Combat the Rise of Loneliness and Improve Mental Wellness in America. Online document at: www.cigna.com/newsroom/ news-releases/2020/cigna-takes-action-to-combat-the-rise-of-loneliness-andimprove-mental-wellness-in-america Accessed January 23, 2020.

2. Geller J, Janson P, McGovern E, et al. Loneliness as a predictor of hospital emergency department use. J Fam Pract 1999;48:801-804.

3. Geller J. Loneliness: An overlooked and costly health risk factor. Minn Med 2000;83:48-51.

4. Geller JS, Dube ET, Cruz GA, et al. Pediatric obesity empowerment model group medical visits (POEM-GMV) as treatment for pediatric obesity in an underserved community. Child Obes 2015;11:638-646.

Jeffrey S. Geller, MD, FP, is Director of Integrative Medicine and Group Programs at Kronos Health, and President of the Integrated Center for Group Medical Visits, in Lawrence, Massachusetts. Dr. Geller is also the cofounder, past president, and current board member for IM4US.

To order reprints of this article, contact the publisher at (914) 740-2100. 